\title{
Burden of Menstrual Pain Measured by Heatmap Visualization of Daily Patient-Reported Data in Japanese Patients Treated with Ethinylestradiol/ Drospirenone: A Randomized Controlled Study
}

This article was published in the following Dove Press journal:

International Journal of Women's Health

\author{
Mikio Momoeda (1D \\ Sayako Akiyama $\mathbb{D}^{2}$ \\ Shigetomo Yamamoto 3 \\ Masami Kondo ${ }^{4}$ \\ Takeshi Fukai ${ }^{4}$ \\ 'Department of Integrated Women's \\ Health, St Luke's International Hospital, \\ Tokyo, Japan; ${ }^{2}$ Market Access, Bayer \\ Yakuhin Ltd., Tokyo, Japan; ${ }^{3}$ Medical \\ Affairs, Bayer Yakuhin Ltd., Osaka, Japan; \\ ${ }^{4}$ Product Development, Bayer Yakuhin \\ Ltd., Osaka, Japan
}

Correspondence: Mikio Momoeda

9-I Akashicho, Chuo-ku, Tokyo I04-8560, Japan

Tel $+8|-3-354|-5|5|$

$\mathrm{Fax}+8 \mathrm{I}-3-5550-2605$

Email momoeda-tky@umin.ac.jp
Purpose: Dysmenorrhea negatively affects women's quality of life and poses a considerable economic burden. A recent study in Japanese patients with dysmenorrhea (NCT01892904) reported a significant reduction in the number of days with menstrual pain after treatment with a flexible extended regimen of ethinylestradiol (EE)/drospirenone (DRSP) compared with a cyclic regimen. However, individual patients' menstrual pain patterns and intensities were not indicated. Heatmapping was used to visualize menstrual pain patterns and intensities by re-evaluating the previously published data from NCT01892904.

Patients and Methods: NCT01892904 was a Phase III, multicenter, randomized, open-label, active-control study of 212 women aged $\geq 20$ years randomized $1: 1$ to receive flexible extended or 28-day cyclic EE/DRSP treatment. Daily pain levels were recorded in patient diaries, and menstrual pain patterns and intensities were visualized using heatmapping. Patients were stratified by baseline dysmenorrhea scores and primary or secondary dysmenorrhea.

Results: The heatmap data demonstrated that EE/DRSP reduced the degree of menstrual pain. Regular peaks of menstrual pain were alleviated in the extended regimen group but were still observed in the cyclic regimen group. While a decrease in the days with menstrual pain was observed in patients with higher baseline dysmenorrhea scores (5-6), those with lower baseline scores (3-4) were more likely to experience lower intensities of menstrual pain. Although pain relief was less likely in patients with secondary dysmenorrhea, those who had lower baseline dysmenorrhea scores (3-4) and received the flexible extended regimen experienced a greater reduction in the number of days with menstrual pain than those who received the cyclic regimen.

Conclusion: Heatmapping effectively visualized the daily burden of menstrual pain in Japanese patients with dysmenorrhea. The analysis using heatmaps suggested that the flexible extended EE/DRSP treatment regimen was more likely to alleviate the regular occurrence of menstrual pain peaks compared with the cyclic regimen.

Keywords: dysmenorrhea, ethinylestradiol, pain measurement, patient-reported outcomes

\section{Plain Language Summary}

Dysmenorrhea can negatively affect women's quality of life and productivity, and ethinylestradiol (EE)/drospirenone (DRSP) may be used to ease the burden of dysmenorrhea. A recent Phase III study of EE/DRSP demonstrated that when compared with a 28-day cyclic regimen, a flexible extended regimen was more effective at alleviating menstrual pain. 
Heatmaps are visual tools that help represent many data points and identify patterns in data. In this analysis, clinical data from the previously published phase III study of EE/DRSP were re-evaluated to create heatmaps and assess differences in menstrual pain patterns and intensities between the two treatment groups. As a result, differences in menstrual pain pattern changes were observed between the groups.

1. Regular peaks of menstrual pain were alleviated in the patients treated with the extended regimen but not in those with the cyclic regimen.

2. Patients with lower baseline dysmenorrhea scores were more likely than those with higher baseline scores to have fewer days with menstrual pain and a greater reduction in the severity of menstrual pain.

3. Patients with primary dysmenorrhea were more likely to experience lower intensities of menstrual pain than those with secondary dysmenorrhea after the treatment.

4. Among the patients with secondary dysmenorrhea, those who had lower baseline dysmenorrhea scores and received the extended regimen experienced a greater reduction in the number of days with menstrual pain compared to those who received the cyclic regimen.

\section{Introduction}

Dysmenorrhea refers to pathological symptoms associated with menstruation. ${ }^{1}$ Primary dysmenorrhea refers to dysmenorrhea that cannot be attributed to a specific cause, and secondary dysmenorrhea is associated with a preexisting gynecological disorder such as endometriosis. Patients present with menstrual pain symptoms such as focal pain (eg, lower abdominal pain, low back pain, and pain radiating to the legs) with the onset of menses. ${ }^{2}$ Some patients also experience nausea, headache, dizziness, insomnia, anxiety/irritability, diarrhea, and depression. ${ }^{2}$

Dysmenorrhea not only affects women's health due to pain during menstruation, but has also been shown to have a negative impact on their quality of life (QOL). ${ }^{3,4}$ According to a comprehensive review including large community-based samples of women, dysmenorrhea affects $16 \%$ to $91 \%$ of women during menstruation, while two to $29 \%$ of cases are classified as severe. ${ }^{5}$ A strong association has been reported between severity of dysmenorrhea and poor school attendance and performance. ${ }^{6,7}$

Dysmenorrhea also poses a considerable economic burden, given its impact on work productivity and healthcare resource utilization. A survey conducted in over 20,000 women in Japan revealed that $17.2 \%$ of the women experienced work productivity loss due to menstrual symptoms within the past three months. ${ }^{8}$ In the survey, an annual economic burden of menstrual symptoms in the Japanese female population was estimated to be 683 billion Japanese yen. ${ }^{8}$ A previous retrospective study of Japanese claims data found that direct costs in the year after diagnosis for women with dysmenorrhea were two to three times higher than those for women without dysmenorrhea. $^{9}$

According to the guidelines of the Japan Society of Obstetrics and Gynecology, treatment of dysmenorrhea should consist of analgesics, such as non-steroidal antiinflammatory drugs, and hormonal contraceptives (such as low-dose estrogen/progestin or levonorgestrel-releasing intrauterine systems). ${ }^{10}$ A study in women with dysmenorrhea suggested that a flexible extended regimen of ethinylestradiol (EE)/drospirenone (DRSP) administered for up to 120 days significantly reduced the number of days with menstrual pain compared with a standard 28-day cyclic regimen (a mean of 3.4 fewer days with dysmenorrheic pain). ${ }^{11}$ However, limitations of that study included that the results did not indicate individual patients' menstrual pain patterns and daily pain levels, but rather combined all patient data. $^{11}$

Usual methods for measuring quantitative data are not necessarily suited to representing qualitative data, such as pain. A heatmap is one visualization method that shows the magnitude of values using shades of color and may provide an effective method for measuring individual pain data. By arranging the colors such that they do not overlap, it is possible to maintain visibility and read all samples widely and objectively. In healthcare settings, heatmaps are used to provide clear visual illustrations of utilization rates of certain tests among different healthcare providers, ${ }^{12}$ and to identify differences in the density of important health and social indicators among neighborhoods to inform healthcare decision-making. ${ }^{13}$ They are also used to visualize high-density data, for example, to detect patterns from chemistry data involving many thousands of different data measurements, ${ }^{14}$ or to map genetic determinants. ${ }^{15}$ Because heatmaps represent an effective method of visualizing patterns, heatmapping was used in this analysis to investigate differences in menstrual pain patterns and degrees of pain between the two EE/DRSP treatment groups (flexible extended and 28-day cyclic regimens), based on re-evaluated data from the previously published phase III study of EE/DRSP in Japanese patients with dysmenorrhea, ${ }^{11}$ and to evaluate the effect of EE/DRSP using a heatmap visualizing daily menstrual pain. 


\section{Materials and Methods}

\section{Study Design}

This was a phase III, multicenter, randomized, open-label, active-control, parallel-group study (BAY 86-5300/16114) sponsored by Bayer Yakuhin, Ltd. (Osaka, Japan), and conducted at eight facilities in Japan. ${ }^{11}$ The study consisted of a 24-week treatment phase followed by a 28 -week long-term safety study. Patients considered eligible for inclusion were aged $\geq 20$ years and had primary or secondary dysmenorrhea as shown by a baseline dysmenorrhea score of $\geq 3$ (the sum of two sub-scores describing severity of dysmenorrhea and use of analgesics [Table 1]; maximum score is 6) in each of the two menstrual cycles during the baseline observation period. The severity of dysmenorrhea sub-score ranged from 0 (none) to 3 (severe: necessary to stay in bed all day; unable to do any work, including schoolwork or housework), and the use of analgesics score ranged from 0 (none) to 3 (severe: used analgesics for three or more days during the previous [or current] menstrual period).

Patients were randomized 1:1 to either the flexible extended regimen group or the 28-day cyclic regimen group. After confirming two menstrual cycles (Visits 2

Table I Baseline Dysmenorrhea Score (Sum of Two Sub-Scores)

\begin{tabular}{|c|c|c|c|}
\hline Sub-Score & Severity & Definition & Score \\
\hline \multirow[t]{4}{*}{$\begin{array}{l}\text { Severity of } \\
\text { dysmenorrhea } \\
\text { sub-score }\end{array}$} & None & $\begin{array}{l}\text { No impairment of work, } \\
\text { including schoolwork and } \\
\text { housework }\end{array}$ & 0 \\
\hline & Mild & $\begin{array}{l}\text { Slight impairment of work, } \\
\text { including schoolwork and } \\
\text { housework }\end{array}$ & 1 \\
\hline & Moderate & $\begin{array}{l}\text { Desire to lie down to rest. } \\
\text { Discomfort interferes with } \\
\text { work, including schoolwork } \\
\text { and housework }\end{array}$ & 2 \\
\hline & Severe & $\begin{array}{l}\text { Necessary to stay in bed } \\
\text { all day. Discomfort causes } \\
\text { inability to work, including } \\
\text { schoolwork and housework }\end{array}$ & 3 \\
\hline \multirow{4}{*}{$\begin{array}{l}\text { Use of } \\
\text { analgesics sub- } \\
\text { score }\end{array}$} & None & No pain & 0 \\
\hline & Mild & $\begin{array}{l}\text { Use of analgesics for I day } \\
\text { during the previous (or } \\
\text { current) menstrual period }\end{array}$ & 1 \\
\hline & Moderate & Use of analgesics for 2 days & 2 \\
\hline & Severe & $\begin{array}{l}\text { Use of analgesics for } 3 \text { or } \\
\text { more days }\end{array}$ & 3 \\
\hline
\end{tabular}

and 3) during the baseline observation period (from Visit 1 to the start of study drug administration), the study drug was provided at Visit 4 and administration was started from the next menstruation. During the treatment period (from the start of study drug administration to Visit 10), administration of the drug by the flexible extended regimen or 28-day cyclic regimen was continued for 24 weeks until Visit 10. Patients randomized to the flexible extended regimen received one tablet of oral EE $20 \mu \mathrm{g} / \mathrm{DRSP} 3 \mathrm{mg}$ every day for at least 24 days and up to 120 days, followed by a four-day interval without study drug administration (cycle of 28 up to 124 days). In the event of three consecutive days of spotting and/or bleeding during days 25 -120 of the intake cycle, a four-day interval was given, followed by the start of the next intake cycle. Patients randomized to the 28-day cyclic regimen received one tablet of EE $20 \mu \mathrm{g} / \mathrm{DRSP} 3 \mathrm{mg}$ at the same time every day for 24 days, followed by four days of placebo tablet administration. Patients in this group received six cycles of treatment during the treatment phase.

The phase III study was conducted in accordance with the ethical principles that have their origin in the Declaration of Helsinki and the International Conference on Harmonization guideline E6: Good Clinical Practice and met all local legal and regulatory requirements. The protocol and all protocol amendments were reviewed and approved by each study site's Independent Ethics Committee/Institutional Review Board. All patients provided written informed consent before entering the study. The study was registered at ClinicalTrials. gov (NCT01892904).

\section{Pain Measurement}

The pain measurement methodology, which is a combined assessment of the degree of menstrual pain and the level of interference to daily activities was previously published (hereinafter "menstrual pain score"). ${ }^{11}$ In brief, from Day 25 of treatment, the patients measured the degree of menstrual pain (Table 2) on a daily basis and recorded their scores in eDiary, an electronic platform that allows patients to report outcomes, regardless of the state of menstrual bleeding (withdrawal bleeding). They rated the menstrual pain as mild (no need for analgesics and no impairment of daily activities), moderate (requiring analgesics, but pain was effectively relieved during most cycles; discomfort interferes with usual daily activities), or severe (required analgesics, but achieved no consistent relief from analgesia; discomfort causes inability to work or perform usual activities). 
Table 2 Evaluation Criteria for Menstrual Pain and the Coloration Used for the Heatmap

\begin{tabular}{|l|l|l|}
\hline Severity & Definition & $\begin{array}{l}\text { Coloration } \\
\text { Used for } \\
\text { Heatmap }\end{array}$ \\
\hline None & No pain. & White \\
\hline Mild & $\begin{array}{l}\text { No need for analgesics and no } \\
\text { impairment of daily activities. }\end{array}$ & Light orange \\
\hline Moderate & $\begin{array}{l}\text { Moderate menstrual pain requiring } \\
\text { analgesics. The pain is relieved by } \\
\text { taking analgesics. Discomfort } \\
\text { interferes with usual daily activities. }\end{array}$ & Orange \\
\hline Severe & $\begin{array}{l}\text { Advanced menstrual pain requiring } \\
\text { analgesics. The pain may not be } \\
\text { relieved by taking analgesics. } \\
\text { Discomfort causes inability to work } \\
\text { or perform usual activities. }\end{array}$ & Dark orange \\
\hline
\end{tabular}

\section{Present Analysis and Heatmapping Methodology}

All the eligible patients who received at least one dose of the study drug were included in this heatmapping analysis. The time period applied in the analysis was from 70 days prior to the start of study drug administration to Day 168 , the end of study drug administration. In addition, a quantitative assessment of the intensity of menstrual pain was conducted for a 140-day period from Day 25.

To visualize menstrual pain patterns and intensities among the two treatment groups, the severity of menstrual pain was displayed in color (Table 2), with the patients on the vertical axis and time on the horizontal axis. Patients with dysmenorrhea scores 3-4 and 5-6 at baseline were placed as subgroups on the top and bottom of the map, respectively, and the patients in each subgroup were ordered by the number of days they had menstrual pain prior to the start of study drug administration. Heatmaps for each subgroup of primary and secondary dysmenorrhea were also created, and the patients in each subgroup, dysmenorrhea scores 3-4 and 5-6 at baseline, were ordered by the number of days they had menstrual pain in the heatmap. There were some missing data because data collection relied on patient self-reporting (3.3\% and $3.0 \%$ in the flexible extended regimen group and 28-day cyclic regimen group, respectively). The missing values were displayed in light gray color, and patients with substantial missing values were arranged above or below the graph.
The numbers of patients who experienced severe pain at least once in the period between Days 25 and 165 were tabulated by treatment group and by subgroups defined by the baseline dysmenorrhea score and were evaluated. Considering that menstrual pains were recurrent events in the same patient over time, the incidence rate was calculated as the number of events across the patients divided by the total number of days assessed. In addition, the marginal mean and rate model was used to estimate the mean cumulative function (MCF) and the rate ratio (RR) with the $95 \%$ confidence interval constructed from the robust sandwich estimate of the covariance matrix. ${ }^{16}$ The same analysis was performed by the subgroups defined by the baseline dysmenorrhea score (3-4 or 5-6). All the analyses were conducted by SAS ver 9.2. Due to the exploratory nature of the analysis conducted after the completion of the study, all analyses were descriptive.

\section{Results}

\section{Patients}

In the phase III study of EE/DRSP, ${ }^{11} 212$ patients received the study medication. Therefore, the full analysis set consisted of 212 patients, 105 randomized to the flexible extended regimen group and 107 to the 28-day cycle regimen group. Patient baseline characteristics were previously described. ${ }^{11}$ In brief, all patients were Asian, and had a mean \pm standard deviation (SD) age of $29.7 \pm 6.4$ years, mean body mass index $20.6 \pm 2.5 \mathrm{~kg} / \mathrm{m}^{2}$, and mean menstrual cycle length SD $29.2 \pm 4.5$ days. The proportion of the patients with primary dysmenorrhea was $71.4 \%$ and $68.2 \%$ in the flexible extended regimen group and cyclic regimen group, respectively. Of the 64 patients with secondary dysmenorrhea, 42 patients had endometriosis, 20 had uterine fibrosis, and 11 had adenomyosis. The proportion of the patients with baseline dysmenorrhea scores of 3-4 was $36.2 \%$ and $43.0 \%$ in the flexible extended regimen group and the cyclic regimen group, respectively, while that with baseline dysmenorrhea scores of 5-6 was $63.8 \%$ and $57.0 \%$ in the flexible extended regimen group and the cyclic regimen group, respectively.

\section{Heatmap Visualization Using the Full Analysis Set}

There was a difference in the menstrual pain patterns after administration of the study drug between the flexible extended regimen group (Figure 1A) and the 28-day cyclic regimen group (Figure 1B) as visualized on the heatmap. 
A

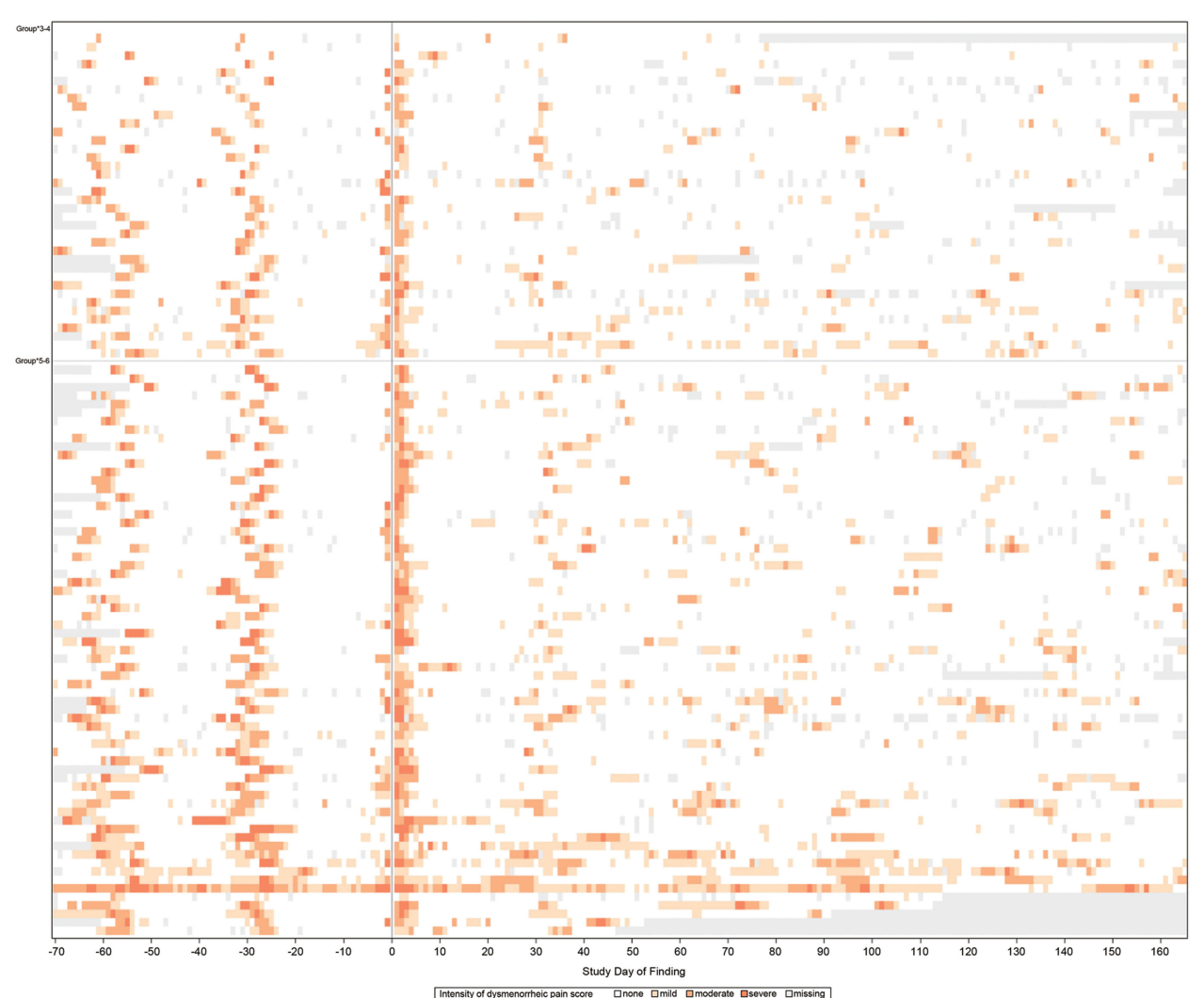

B

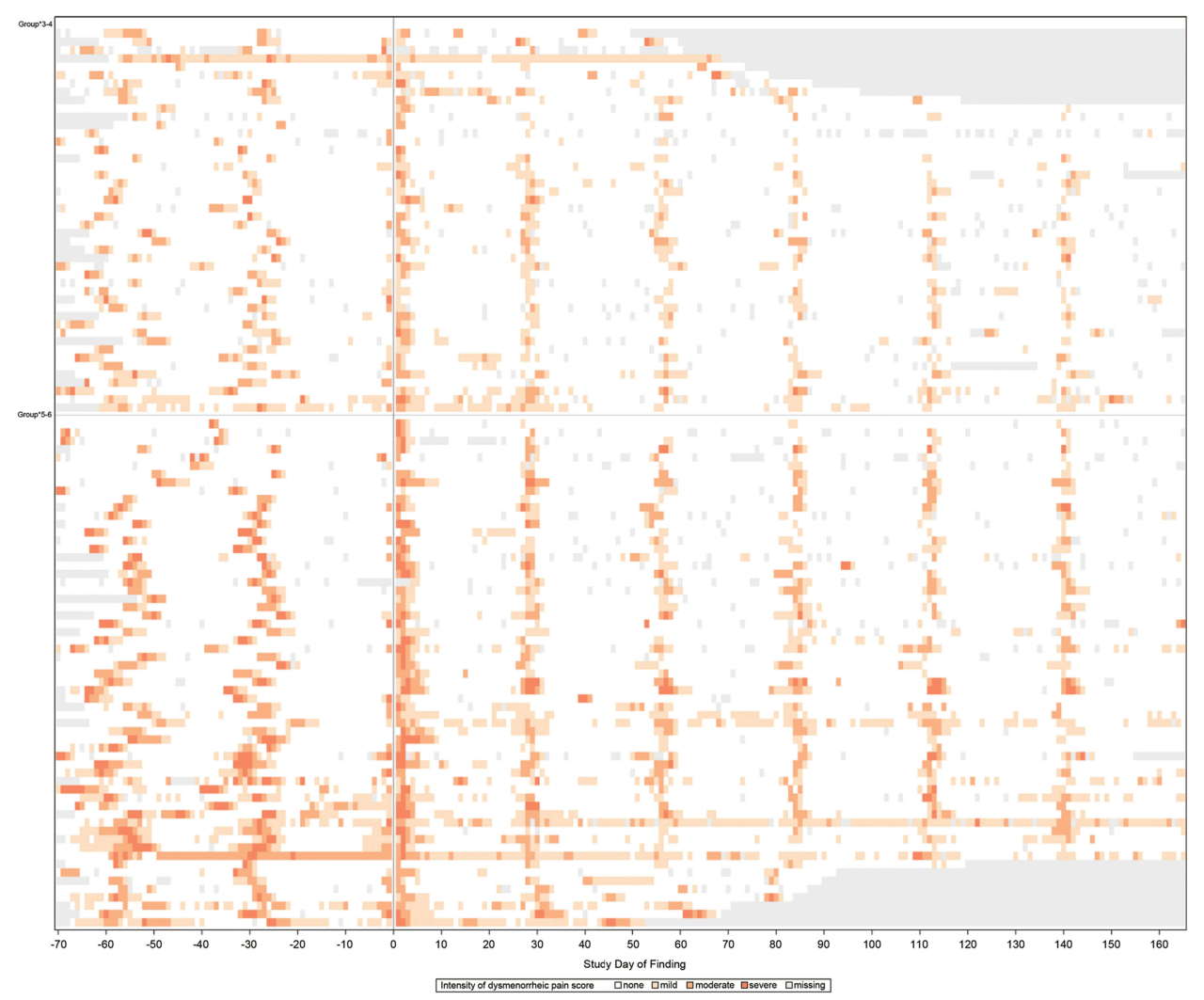

Figure I Overall menstrual pain scores over 24 weeks in the full analysis set following treatment with the flexible extended regimen (A) and the 28-day cyclic regimen (B), as shown with heatmapping. The reference line at Day 0 indicates the first day of study drug administration. Lower baseline dysmenorrhea scores (3-4) are shown in the top half of the heatmap, and higher baseline dysmenorrhea scores (5-6) in the bottom half. 
In both groups, two or more peaks of pain thought to be due to menstruation were observed before the start of study drug administration. After the start of administration, the two treatment groups presented different peak patterns of menstrual pain. While the degree of menstrual pain appeared to be alleviated in both groups initially, regular peak patterns of menstrual pain were still observed in the 28-day cyclic regimen group but not in the flexible extended regimen group. The map suggested that severe menstrual pain in the flexible extended regimen group was more likely to be alleviated after study drug administration, compared with the cyclic regimen group. Over the 140 days of the evaluation period, patients treated with the flexible extended regimen reported on average 3.4 fewer days with menstrual pain than those with the 28-day cyclic regimen $(95 \% \mathrm{CI}-6.5$ to $-0.3 ; \mathrm{P}=0.03)$. Patients with low baseline dysmenorrhea scores (3-4), especially those in the flexible extended regimen group, tended to have a lower degree of menstrual pain at the peak of pain after study drug administration compared with those in the 28-day cyclic regimen group. Although patients with high baseline dysmenorrhea scores (5-6) experienced lower intensities of menstrual pain after administration, when compared with patients with low baseline scores, they tended to continue to experience menstrual pain after administration, and the peak post-administration pain levels were relatively higher in the cyclic regimen group compared to the flexible extended regimen group.

\section{Exploratory Analysis}

The percentage of the patients who experienced severe menstrual pain more than once was $15.2 \%(16 / 105)$ in the flexible extended regimen group and $27.1 \%(29 / 107)$ in the 28-day cyclic regimen group (Table 3). Similarly, in the analysis by baseline score, the proportion of the patients who experienced severe menstrual pain tended to be lower in the flexible extended regimen group than in the cyclic regimen group (Table 3 ).

The percentage of the days with severe menstrual pain in the total number of the days assessed was $0.23 \%(32 / 13,884)$ in the flexible extended regimen group and $0.58 \%(78 / 13,377)$ in the 28-day cyclic regimen group (Table 4), and the MCF for severe menstrual pain for both groups was plotted (Figure 2); the rate ratio (RR) for severe menstrual pain in the 28-day cyclic regimen group vs the flexible extended regimen group was 2.478 (95\% CI: 1.2, 5.2) (Table 4). The analysis for severe menstrual pain was also performed by baseline dysmenorrhea score; the results of the heatmapping and the RR for the 28-day
Table 3 Number of Patients with Menstrual Pain by Intensity

\begin{tabular}{|l|l|l|l|l|l|}
\hline $\begin{array}{l}\text { Dysmenorrhea } \\
\text { Score at } \\
\text { Baseline }\end{array}$ & & $\begin{array}{l}\text { Flexible } \\
\text { Extended } \\
\text { Regimen }\end{array}$ & & $\begin{array}{l}\text { 28-Day } \\
\text { Cyclic } \\
\text { Regimen }\end{array}$ & \\
\hline Total & $\mathrm{n}$ & 105 & $100.0 \%$ & 107 & $100.0 \%$ \\
& None & 0 & & $\mathrm{I}$ & $0.9 \%$ \\
& Mild & 31 & $29.5 \%$ & 20 & $18.7 \%$ \\
& Moderate & 58 & $55.2 \%$ & 57 & $53.3 \%$ \\
& Severe & 16 & $15.2 \%$ & 29 & $27.1 \%$ \\
\hline $3-4$ & $\mathrm{n}$ & 38 & $100.0 \%$ & 46 & $100.0 \%$ \\
& None & 0 & & 1 & $2.2 \%$ \\
& Mild & 16 & $42.1 \%$ & 15 & $32.6 \%$ \\
& Moderate & 18 & $47.4 \%$ & 24 & $52.2 \%$ \\
& Severe & 4 & $10.5 \%$ & 6 & $13.0 \%$ \\
\hline $5-6$ & $\mathrm{n}$ & 67 & $100.0 \%$ & 61 & $100.0 \%$ \\
& None & 0 & & 0 & \\
& Mild & 15 & $22.4 \%$ & 5 & $8.2 \%$ \\
& Moderate & 40 & $59.7 \%$ & 33 & $54.1 \%$ \\
& Severe & 12 & $17.9 \%$ & 23 & $37.7 \%$ \\
\hline
\end{tabular}

cyclic regimen group vs the flexible extended regimen are shown in Table 4.

\section{Heatmap Visualization by Dysmenorrhea Type}

The pain scores after treatment with the flexible extended regimen and the 28-day cyclic regimen according to dysmenorrhea subgroup type using heatmapping are shown in Figure 3 (primary dysmenorrhea) and Figure 4 (secondary dysmenorrhea).

When assessed separately by primary and secondary dysmenorrhea, patients with low baseline dysmenorrhea scores in both subgroups were likely to gain some pain relief. Although the secondary dysmenorrhea patients, compared with the primary dysmenorrhea patients, tended to be less likely to experience pain relief, the secondary dysmenorrhea patients treated with the flexible extended regimen, compared with those with the 28-day cyclic regimen, experienced a greater reduction in the number of days with menstrual pain (mean \pm SD: $13.8 \pm 10.7$ versus $17.8 \pm 13.4$ days), particularly those with low baseline dysmenorrhea scores.

\section{Discussion}

Dysmenorrhea is considered to affect almost all menstruating women at some point in their lives, with almost onethird reporting severe menstrual pain. ${ }^{5}$ Therapeutic improvements are needed to reduce the incidence and severity of dysmenorrhea, its impact on individual women's $\mathrm{QOL}^{3,4}$ and its wider socioeconomic impact. ${ }^{7,8}$ 
Table 4 Rate Ratios for Severe Menstrual Pain for the 28-Day Cyclic Regimen vs Flexible Extended Regimen According to Baseline Dysmenorrhea Score

\begin{tabular}{|c|c|c|c|c|}
\hline $\begin{array}{l}\text { Dysmenorrhea } \\
\text { Score at Baseline }\end{array}$ & Treatment Group & $\begin{array}{l}\text { Total Number of } \\
\text { Days Assessed }\end{array}$ & $\begin{array}{l}\text { Total Number of } \\
\text { Days with Severe } \\
\text { Pain Score (\%) }\end{array}$ & $\begin{array}{l}\text { RR:28-Day Cyclic Regimen } \\
\text { Group/Flexible Extended } \\
\text { Regimen }(95 \% \mathrm{Cl})\end{array}$ \\
\hline Total & $\begin{array}{l}\text { Flexible extended regimen group } \\
\text { 28-day cyclic regimen group }\end{array}$ & $\begin{array}{l}13,884 \\
13,377\end{array}$ & $\begin{array}{l}32(0.23) \\
78(0.58)\end{array}$ & $2.478(1.182-5.196)$ \\
\hline $3-4$ & $\begin{array}{l}\text { Flexible extended regimen group } \\
\text { 28-day cyclic regimen group }\end{array}$ & $\begin{array}{l}5037 \\
5531\end{array}$ & $\begin{array}{l}5(0.10) \\
9(0.16)\end{array}$ & $1.624(0.456-5.778)$ \\
\hline $5-6$ & $\begin{array}{l}\text { Flexible extended regimen group } \\
28 \text {-day cyclic regimen group }\end{array}$ & $\begin{array}{l}8847 \\
7846\end{array}$ & $\begin{array}{l}27(0.31) \\
69(0.88)\end{array}$ & $2.832(I .25 I-6.408)$ \\
\hline
\end{tabular}

Abbreviations: $\mathrm{RR}$, rate ratio; $\mathrm{Cl}$, confidence interval.

In the previously published phase III study of EE/DRSP, the number of days with menstrual pain was 3.4 days fewer in the flexible extended EE/DRSP regimen group compared with the standard cyclic 28-day EE/DRSP regimen group. ${ }^{11}$ In this analysis, data from the phase III study were used to generate heatmaps to present comparative menstrual pain patterns among women with dysmenorrhea treated with the two EE/DRSP regimens and qualitatively visualize changes in menstrual pain levels during treatment. The heatmapping analysis of the daily menstrual pain data enabled us to visualize the degree of menstrual pain and the time-dependent change before and after administration of the study drug.

The heatmap data demonstrated that overall, EE/DRSP reduced the degree of menstrual pain in both regimen

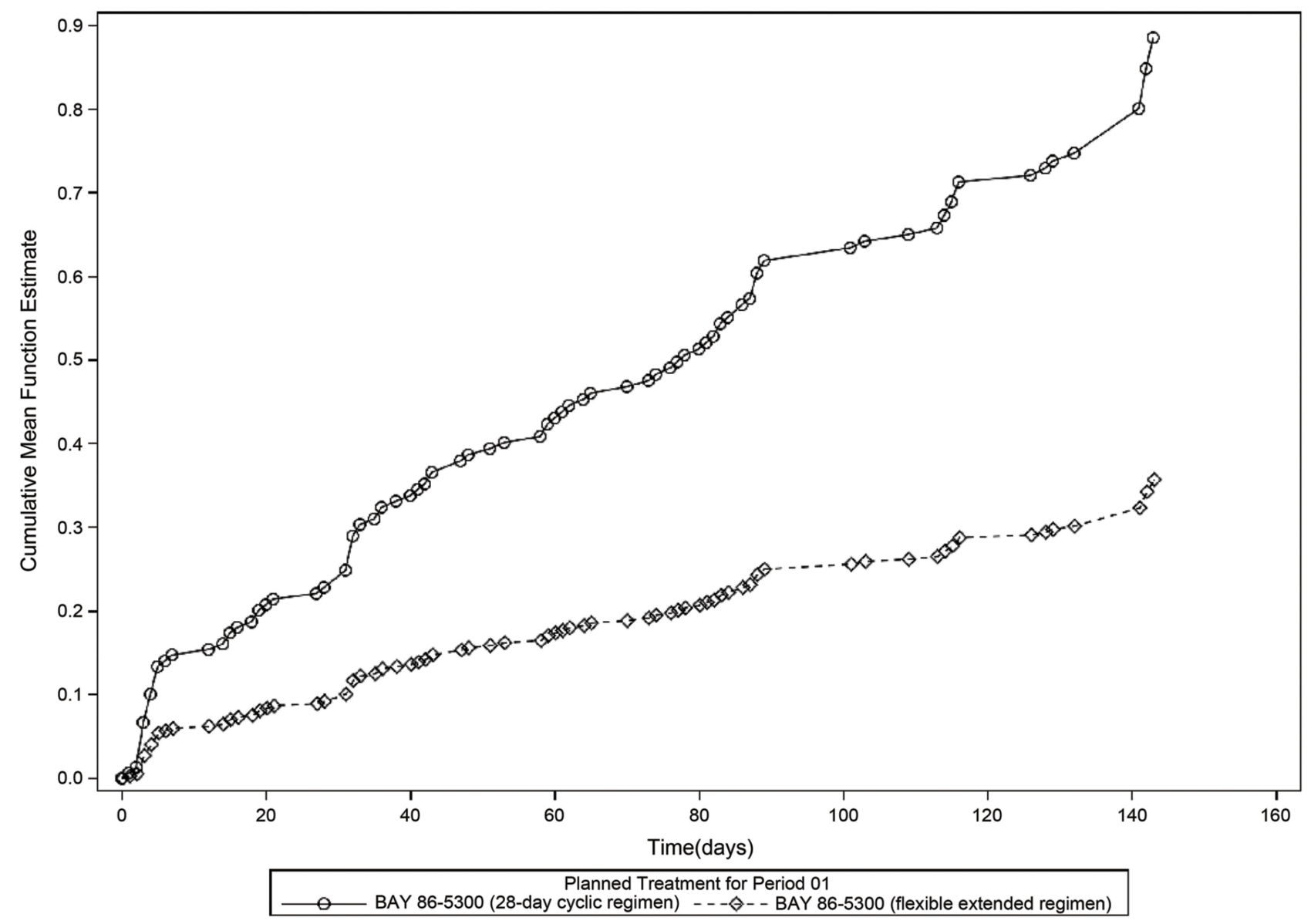

Figure 2 Mean cumulative function of the flexible extended regimen and the 28-day cyclic regimen. 
A

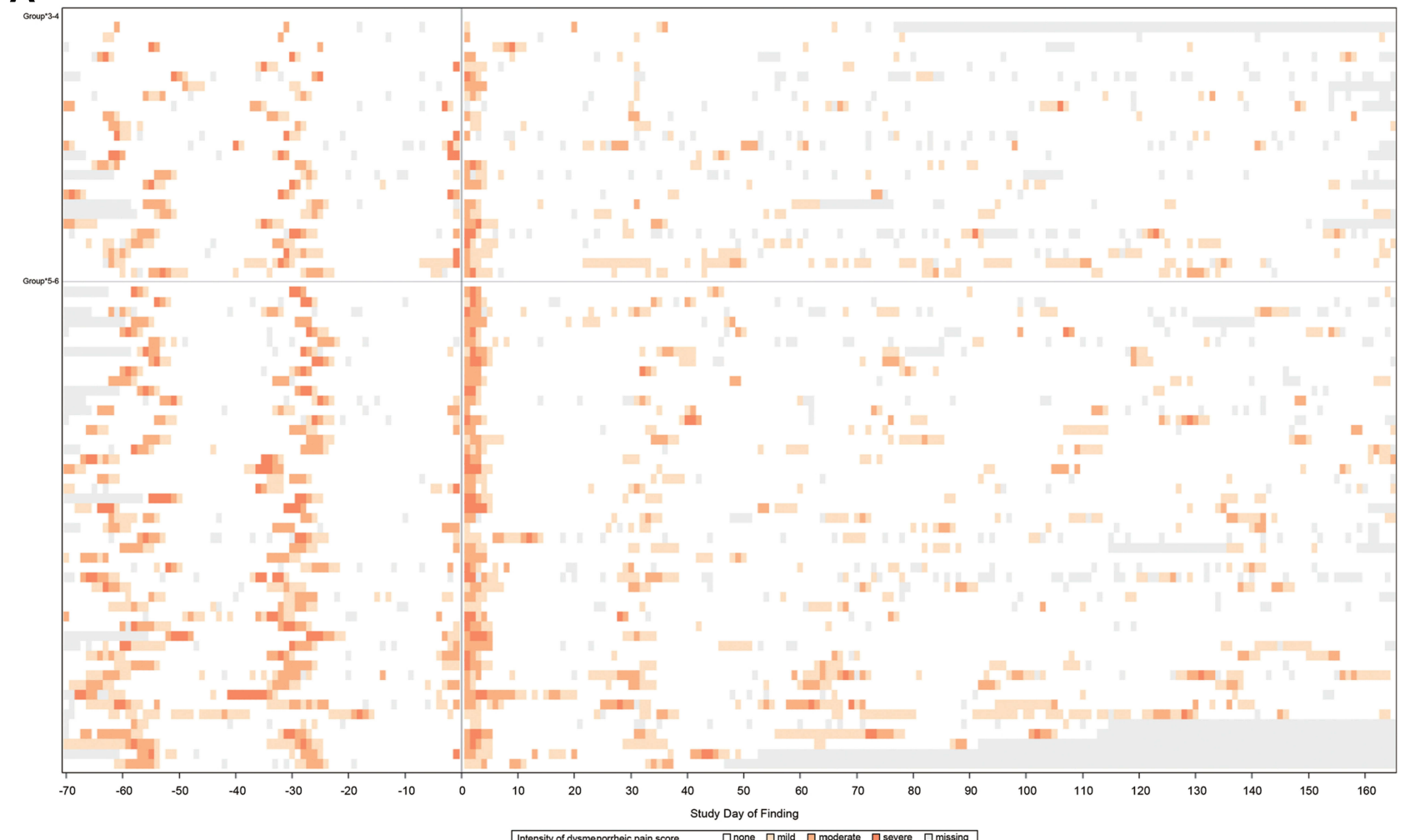

B

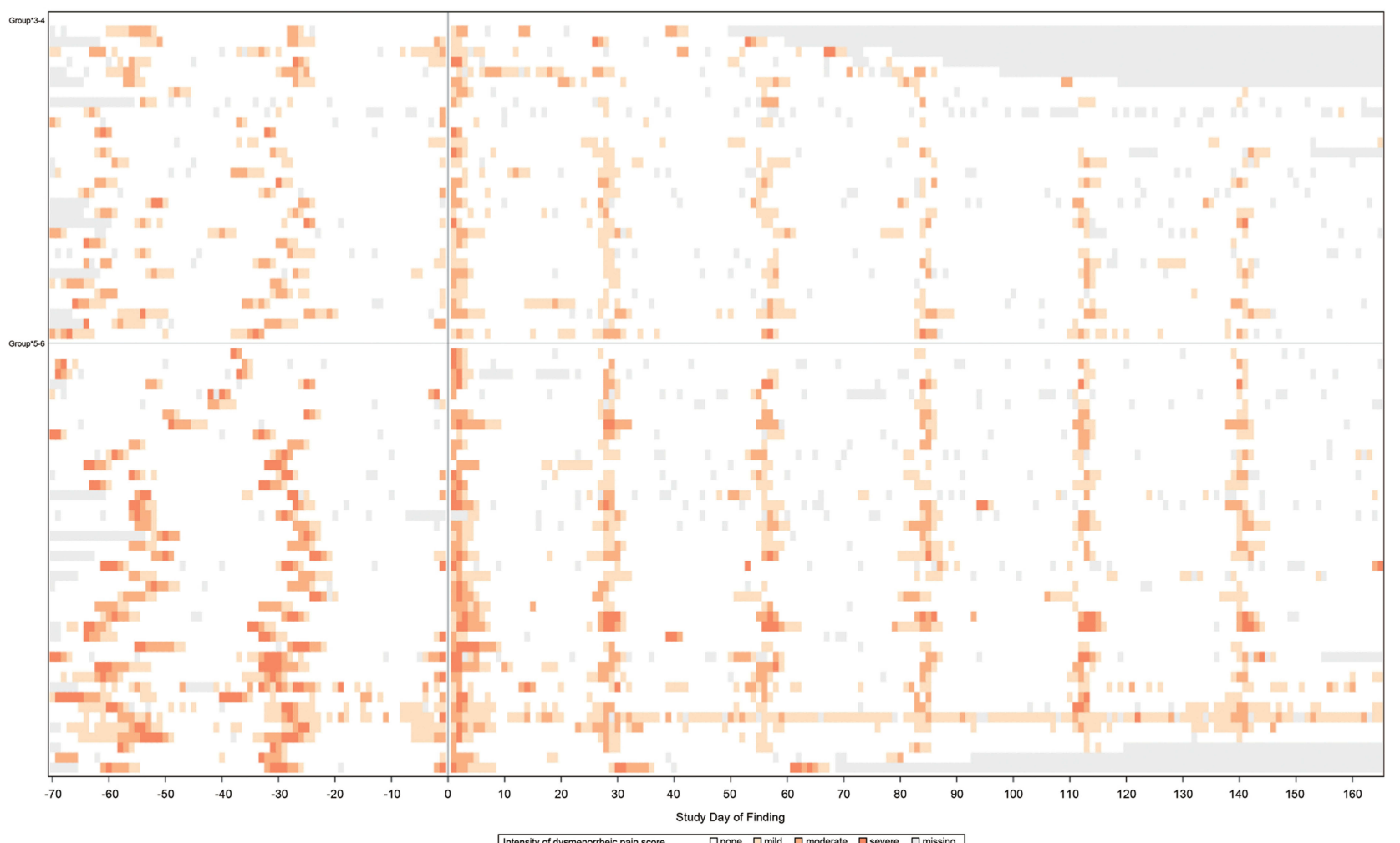

Figure 3 Menstrual pain scores as shown by heatmapping after treatment with the flexible extended regimen (A) or the 28-day cyclic regimen (B) in the group with primary dysmenorrhea. Lower baseline dysmenorrhea scores (3-4) are shown in the top half of the heatmap, and higher baseline dysmenorrhea scores (5-6) in the bottom half. 

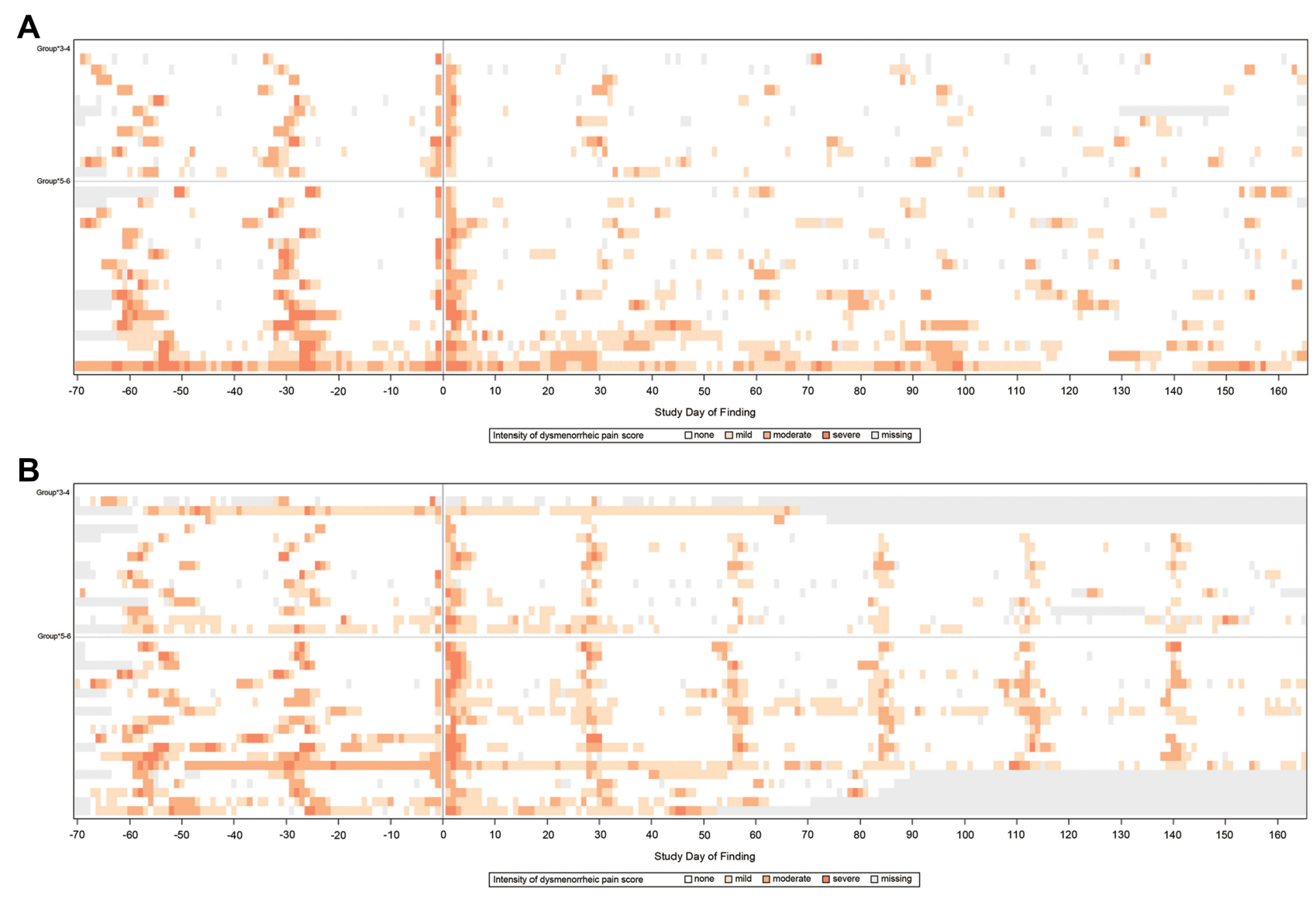

Figure 4 Menstrual pain scores as shown by heatmapping after treatment with the flexible extended regimen (A) or the 28-day cyclic regimen (B) in the group with secondary dysmenorrhea. Lower baseline dysmenorrhea scores (3-4) are shown in the top half of the heatmap, and higher baseline dysmenorrhea scores (5-6) in the bottom half.

groups. The heatmap suggested visually that patients with low baseline dysmenorrhea scores (3-4) were more likely to experience lower intensities of menstrual pain through administration of EE/DRSP, while in patients with high baseline dysmenorrhea scores (5-6), their menstrual pain was not completely alleviated, but some relief could be expected in both groups. The same results were obtained using the RR.

When assessed separately by dysmenorrhea type, patients with low baseline dysmenorrhea scores in both subgroups were also more likely to achieve pain relief to lower intensities. The heatmap suggested that although the secondary dysmenorrhea patients overall, compared with the primary dysmenorrhea patients, tended to be less likely to experience pain relief, those treated with the flexible extended regimen, compared with those with the 28-day cyclic regimen, experienced a greater reduction in the number of days with menstrual pain.

In recent years, due to the explosion of time-series data generated from the Internet of Things (eg, mobile, sensor, and wearable devices), there has been mounting interest in techniques that have the potential to detect and visualize patterns in non-numerical (sensory or subjective) information. Heatmaps are already used for geographical information systems visualizations. ${ }^{17}$ The use of heatmapping is now expanding also within the healthcare field, and the most common uses are to gain information on epidemiological or healthcare utilization patterns and inform public health decisions, ${ }^{12,13}$ or to visualize large datasets. ${ }^{14,15}$ In cancer research, heatmaps are used to identify mitochondrial RNA switching to discover novel tumor biomarkers ${ }^{18}$ and to predict patient survival. ${ }^{19}$ However, heatmapping is not frequently used in the context of clinical studies or to visualize pain scores. Aside from pain scores, heatmapping could potentially be used to visualize subject data related to other areas of women's health, such as volume of menstrual bleeding, and in other areas of study, such as sleep quality. Heatmapping enabled us to obtain new insight that was not apparent in the previous report of the phase III study of EE/DRSP. 
However, there are limitations to this analysis. The number of patients in the secondary dysmenorrhea subgroup was small, being less than one-third of the total. Therefore, when the patients were further divided into subgroups by baseline dysmenorrhea score, the number of each subgroup became even smaller. Missing values in this analysis were placed on the heatmap in a different color from the overall color scheme, and patients with the missing values were placed above and below the map to maintain the pattern visibility.

\section{Conclusions}

The analysis using heatmaps suggested that the flexible extended EE/DRSP treatment regimen was more likely to alleviate the regular occurrence of menstrual pain peaks compared with the 28-day cyclic regimen. The flexible extended regimen appeared to reduce the number of days with menstrual pain and pain levels in the patients with both primary and secondary dysmenorrhea, although the number of the patients in the secondary dysmenorrhea subgroup was small. Heatmapping effectively visualized the daily burden of menstrual pain in the Japanese patients with dysmenorrhea, and may be useful for visualizing individual patient data, including pain scores, in clinical studies, and visually comparing data among different regimens or subgroups.

\section{Abbreviations}

EE/DRSP, ethinylestradiol/drospirenone; RR, rate ratio; $\mathrm{MCF}$, mean cumulative function; QOL, quality of life; $\mathrm{SD}$, standard deviation.

\section{Data Sharing Statement}

Availability of the data underlying this publication will be determined according to Bayer's commitment to the EFPIA/PhRMA "Principles for responsible clinical trial data sharing." This pertains to scope, time point, and process of data access. As such, Bayer commits to sharing, upon request from qualified scientific and medical researchers, patient-level clinical trial data, study-level clinical trial data, and protocols from clinical trials in patients for medicines and indications approved in the US and European Union (EU) as necessary for conducting legitimate research. This applies to data on new medicines and indications that have been approved by the EU and US regulatory agencies on or after January 1, 2014. Interested researchers can use www.clinicalstudydatarequest.com to request access to available datasets and supporting documents from clinical studies to conduct further research that can help advance medical science or improve patient care. Information on the Bayer criteria for listing studies and other relevant information is provided in the "Study sponsors" section of the portal. Data access will be granted to available datasets, protocols, and clinical study reports after approval by an independent scientific review panel. Bayer is not involved in the decisions made by the independent review panel. Bayer will take all necessary measures to ensure that patient privacy is safeguarded.

\section{Ethics Approval and Informed Consent}

The protocol and all protocol amendments were reviewed and approved by each study site's independent ethics committee/institutional review board (Suzuran Clinic, Ikebukuro Clinic, Seijo Kinoshita Hospital, Hayakawa Clinic, New Medical Research System Clinic, Shirokane Ladies' Clinic, Juno Vesta Clinic Hatta, Chayamachi Ladies Clinic). All patients provided written informed consent before entering the study.

\section{Acknowledgments}

Medical writing services were provided by Marion Barnett and Sarah Bubeck, PhD, from Edanz Medical Writing, and sponsored by Bayer Yakuhin, Ltd. The authors would like to thank the patients who participated in the study and the staff members at the study sites who provided care for them. The authors also wish to thank Tetsushi Komori for providing guidance on the data analysis.

\section{Author Contributions}

MM, SA, and TF made contributions to the study conception and design; TF contributed to data analysis. All authors made substantial contributions to the interpretation of data and drafted or critically revised the article for important intellectual content, gave final approval of the version to be published, and agree to be accountable for all aspects of the work.

\section{Funding}

This study was sponsored by Bayer Yakuhin, Ltd., which was involved in the conceptualization and design of the study, interpretation of the study results, and in preparing the manuscript for submission.

\section{Disclosure}

Mikio Momoeda is a paid medical advisor to Bayer Yakuhin, Ltd. for this study and outside of this study. 
Takeshi Fukai, Shigetomo Yamamoto, and Masami Kondo are employees of Bayer Yakuhin, Ltd. and Sayako Akiyama was an employee of Bayer Yakuhin, Ltd. during the conduct of this study. The authors report no other conflicts of interest related to this work.

\section{References}

1. The Japan Society of Obstetrics and Gynecology. Glossary of Gynecological Terms. 4th ed. Tokyo; 2018.

2. Deligeoroglou E. Dysmenorrhea. Ann $N$ Y Acad Sci. 2000;900:237-244. doi:10.1111/j.1749-6632.2000.tb06235.x

3. Unsal A, Tozun M, Arslan G, Calik E. Prevalence of dysmenorrhea and its effect on quality of life among a group of female university students. Ups J Med Sci. 2010;115(2):138-145. doi:10.3109/03009730903457218

4. Wong CL. Health-related quality of life among Chinese adolescent girls with dysmenorrhoea. Reprod Health. 2018;15(1):80. doi:10.1186/ s12978-018-0540-5

5. Ju H, Jones M, Mishra G. The prevalence and risk factors of dysmenorrhea. Epidemiol Rev. 2014;36:104-113. doi:10.1093/epirev/ mxt009

6. Al-Jefout M, Seham AF, Jameel H, et al. Dysmenorrhea: prevalence and impact on quality of life among young adult jordanian females. $J$ Pediatr Adolesc Gynecol. 2015;28(3):173-185. doi:10.1016/j. jpag.2014.07.005

7. Yücel G, Kendirci M, Gül Ü. Menstrual characteristics and related problems in 9- to 18-year-old Turkish school girls. J Pediatr Adolesc Gynecol. 2018;31(4):350-355. doi:10.1016/j.jpag.2018.03.002

8. Tanaka E, Momoeda M, Osuga Y, et al. Burden of menstrual symptoms in Japanese women: results from a survey-based study. $\mathrm{J} \mathrm{Med}$ Econ. 2013;16(11):1255-1266. doi:10.3111/13696998.2013.830974

9. Akiyama S, Tanaka E, Cristeau O, Onishi Y, Osuga Y. Evaluation of the treatment patterns and economic burden of dysmenorrhea in Japanese women, using a claims database. Clinicoecon Outcomes Res. 2017;9:295-306. doi:10.2147/CEOR
10. The Japan Society of Obstetrics and Gynecology. Guideline for Obstetrical Practice; Gynecological Practice in Japan. Tokyo; 2017.

11. Momoeda M, Kondo M, Elliesen J, Yasuda M, Yamamoto S, Harada T. Efficacy and safety of a flexible extended regimen of ethinylestradiol/ drospirenone for the treatment of dysmenorrhea: a multicenter, randomized, open-label, active-controlled study. Int $J$ Womens Health. 2017;9:295-305. doi:10.2147/IJWH

12. Ziemba YC, Lomsadze L, Jacobs Y, Chang TY, Haghi N. Using heatmaps to identify opportunities for optimization of test utilization and care delivery. J Pathol Inform. 2018;9:31.

13. Hardt NS, Muhamed S, Das R, Estrella R, Roth J. Neighborhoodlevel hot spot maps to inform delivery of primary care and allocation of social resources. Perm J. 2013;17(1):4-9. doi:10.7812/TPP/12-090

14. Auman JT, Boorman GA, Wilson RE, Travlos GS, Paules RS. Heat map visualization of high-density clinical chemistry data. Physiol Genomics. 2007;31(2):352-356. doi:10.1152/physiolgenomics.002 76.2006

15. Donaghey J, Thakurela S, Charlton J, et al. Genetic determinants and epigenetic effects of pioneer-factor occupancy. Nat Genet. 2018;50 (2):250-258. doi:10.1038/s41588-017-0034-3

16. Lin DY, Wei LJ, Yang I, Ying Z. Semiparametric regression for the mean and rate functions of recurrent events. $J R$ Stat Soc $B$. 2000;62:711-730. doi:10.1111/1467-9868.00259

17. Netek R, Pour T, Slezakova R. Implementation of heat maps in geographical information system - exploratory study on traffic accident data. Open Geosci. 2018;10:367-384. doi:10.1515/geo-20180029

18. Chen L, Sun H, Wang C, Yang Y, Zhang M, Wong G. miRNA arm switching identifies novel tumour biomarkers. EBioMedicine. 2018;38:37-46. doi:10.1016/j.ebiom.2018.11.003

19. Deng W, Xu T, Xu Y, et al. Survival patterns for patients with resected N2 non-small cell lung cancer and postoperative radiotherapy: a prognostic scoring model and heat map approach. $J$ Thorac Oncol. 2018;13(12):1968-1974. doi:10.1016/j.jtho. 2018.08.2021

\section{Publish your work in this journal}

The International Journal of Women's Health is an international, peerreviewed open-access journal publishing original research, reports, editorials, reviews and commentaries on all aspects of women's healthcare including gynecology, obstetrics, and breast cancer. The manuscript management system is completely online and includes a very quick and fair peer-review system, which is all easy to use Visit http://www.dovepress.com/testimonials.php to read real quotes from published authors. 